\title{
LAS IDEAS ECONÓMICO-SOCIALES DE AMÉRICA LATINA Y EL CARIBE EN PAKISTÁN: RECEPCIÓN Y REELABORACIÓN (1965-1980)1
}

\author{
SOCIO-ECONOMIC IDEAS OF LATIN AMERICA AND \\ THE CARIBBEAN IN PAKISTAN: RECEPTION AND \\ RE-ELABORATION (1965-1980)
}

\section{EDUARDO DEVÉS-VALDÉS ${ }^{2}$}

\section{RESUMEN}

El objetivo del artículo es presentar las recepciones y reelaboraciones en Pakistán del pensamiento económico-social producido en América Latina y el Caribe. Se presentan varios autores que en Pakistán recibieron y reelaboraron las ideas económico-sociales latinoamericanas: los casos de M. A. Mannan y de Mahbub ul-Haq, así como el contexto eidético o medioambiente intelectual en que tales ideas fueron recibidas y reelaboradas. Se determinan cuáles fueron las ideas, autores y obras recepcionados por los autores pakistaníes. Luego, se muestran las diferencias entre las formas en que los pakistaníes recepcionaron a los autores de América Latina y el Caribe y las maneras en que se hibridó el pensamiento de ALC con otras trayectorias eidéticas, apuntando a elaborar formulas que correspondan a "tipos ideales" de hibridación.

${ }^{1}$ Este artículo es producto del proyecto Fondecyt No 1070104. Se ha realizado sobre la base de investigaciones efectuadas en la Jawaharlal Nerhu University y en el Jawaharlal Nerhu Museum de Nueva Delhi, y agradezco particularmente al profesor Shyama Prasad Ganguly; en CODESRIADakar, agradezco particularmente a la profesora Sokhna Gueye; en IDEP y Universidad Ch. A. Diop de Senegal, agradezco particularmente al profesor Amadou Ndoye; en el "Centro de Estudos Afro-Asiáticos” de la Universidad Cándido Mendes de Río de Janeiro, agradezco particularmente al profesor Beluce Belluci; en la Universidad de Puerto Rico en Río Piedras; en la biblioteca de la CEPAL en Santiago de Chile, a través de búsquedas en Internet y de la correspondencia con los propios autores estudiados, como Susantha Goonatilake, cuando ello ha sido posible.

${ }^{2}$ Investigador del Instituto de Estudios Avanzados (IDEA) de la Universidad de Santiago de Chile, Román Díaz 89, Providencia, Santiago, Chile. Tel: 56-2-7181360.E-mail: eduardo.deves@ usach.cl . Todos los textos citados que aparecen en la bibliografía en otros idiomas han sido traducidos por el autor. 
Palabras clave: Pensamiento periférico, ciencias económico-sociales, socialismo islámico, economía para la liberación, hibridaciones eidéticas.

\begin{abstract}
The main objective of this article is to present the reception and re-elaboration of Latin-American social and economic thought in Pakistan. Some authors that have worked with Latin-American ideas are introduced, such as M. A. Mannan and Mahbub ul-Haq, as well as the eidetic context or intellectual environment in which these ideas were received and re-elaborated. First of all, the article defines which were the ideas, authors and works considered by the Pakistani authors; secondly, it shows the differences in the ways each Pakistani writer received the ideas of Latin American and Caribbean authors and the ways that Latin American and Caribbean thought was "hybridated" in relation to other eidetic trajectories with the aim of elaborating formulas that would correspond to "ideal types" of hybridations.
\end{abstract}

Keywords: Peripheral thought, social and economic sciences, Islamic socialism, economy of liberation, eidetic hybridations.

Recibido: 18.01.10. Aceptado: 21.09.11.

\title{
1. INTRODUCCIÓN
}

1 .1. ESTE ARTÍCULO se ocupa de las relaciones intelectuales entre América Latina y el Caribe (ALC) y Asia (ver Devés y Melgar-Bao, 2005) y particularmente de la recepción y reelaboración en Pakistán (Pk), de algunos aspectos de las ciencias económico-sociales producidas en ALC durante los 60 y 70. Se trata apenas de una introducción a estos asuntos, a partir de investigaciones realizadas en la Jawaharlal Nerhu University y en el Jawaharlal Nerhu Museum de Nueva Delhi, en el "Centro de Estudos Afro-Asiáticos" de la Universidad Candido Mendes de Río de Janeiro, en la Universidad de Puerto Rico en Río Piedras, en la biblioteca de la CEPAL en Santiago de Chile y a través de búsquedas en Internet. Se ha utilizado únicamente material publicado, de circulación internacional y en lenguas de origen europeo, como consta en la bibliografía.

Para quien se ocupa de estudios eidológicos sería muy bello poder formular una hipótesis, sobre la presencia y reelaboración de las ciencias económico-sociales de ALC en Pk, en la cual se enunciara algo así como: "El pensamiento de ALC fue recibido en Pk en el marco del pensamiento islámico, heredado de Avicena y reelaborado mediante cruzamientos con 
el deobandismo ${ }^{3}$, generando una suerte de desarrollismo islámico socialista...". De no ser posible una hipótesis de este calibre sería interesante, al menos, sugerir que "el pensamiento económico-social de ALC se cruzó con las formulaciones independentistas de Muhammad Jinnah y con el panislamismo de Muhammad Iqbal, los más importantes fundadores de $\mathrm{Pk}$, produciendo síntesis que reelaboradas en el marco del...". Pero, las hipótesis que se formularán a continuación requieren de menos vuelo para la fantasía de quienes imaginarían exóticos viajes eidéticos, que más se acercarían a la eidología-ficción, y donde los clichés sobre el subcontinente indo-pakistaní (SIP) querrían ver a Arthur Lewis, a Raúl Prebisch o a Celso Furtado generando descendencia con hibridaciones védicas y donde los estudios pudieran abocarse a la lectura de algo así como un kamasutra eidético, concebido en las mil y una noches, en una proliferación fantástica de sofisticados cruzamientos en cadena y sin fin.

Lamentablemente no se podrá deleitar a quienes se interesan por estos asuntos, como se hubiera deseado. Las hibridaciones que se expondrán son menos brillantes, las distancias geneidéticas entre las tendencias son menores, los cruzamientos en consecuencia menos espectaculares y los descendientes menos fenomenales. Se estudian los contactos entre intelectualidades periféricas "modernas", que utilizan marcos conceptuales y paradigmas similares. Es decir, los aportes de este trabajo pueden ubicarse mucho más en relación a aquello que la comunidad de estudios eidéticos ignora que respecto al exotismo de las hibridaciones descubiertas. En buena medida, los autores que se encuentran, aunque por cada lado puedan compartir algunos elementos con sus respectivas culturas ancestrales, generalmente, han realizado la "separación entre ciencia y fe", por decirlo de algún modo. Incluso si, en verdad, ello no es absoluto y quien lea no dejará de llevarse algunas sorpresas.

${ }^{3}$ Asumiendo la necesidad de una producción industrial de artículos, se ha optado por la formulación que consiste en subir a Internet una gran cantidad de piezas, llamémoslas "accesorias", de modo de no tener que incorporarlas a cada artículo, especialmente si van a ser publicados en papel. Ello implicaría un encarecimiento sustancial, por el crecimiento del texto. Los términos que corresponden a personas, escuelas de pensamiento, instituciones y redes, pueden consultarse en Atlas del pensamiento en www.umbral.uprrp.edu. Allí podrán encontrarse miles de otras informaciones y miles más de conexiones para quienes deseen profundizar en estos temas. El proyecto Atlas del pensamiento, como su nombre lo indica, apunta a exhibir geográficamente el pensamiento, particularmente de las regiones periféricas, durante los 3 últimos siglos. En el impreso se entregan únicamente las referencias más necesarias. Los nombres de los autores se han escrito tal como aparecen en los textos de su autoría.

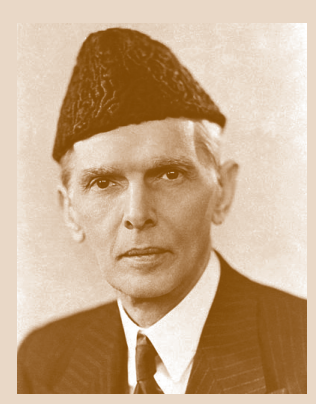

M. A. Jinnah

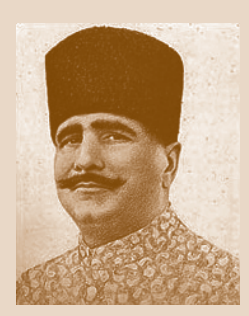

M. Iqbal

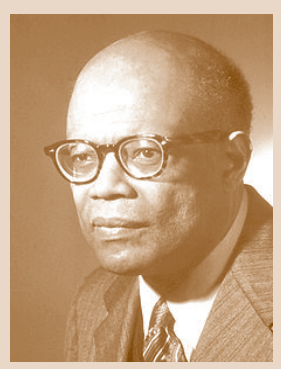

A. Lewis

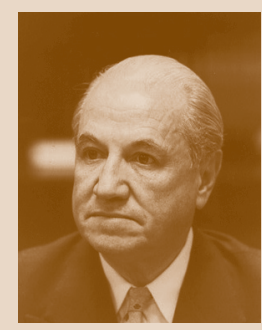

R. Prebisch 
1.2. El pensamiento de la intelectualidad angloparlante anterior a la independencia (1947) en el SIP fue eminentemente político-cultural, aunque hubo algunos autores que abordaron cuestiones específicamente económicas. Al no tener que gestionar sus respectivas sociedades, debido a la presencia colonial, las elites se abocaron a "generar identidad e independencia" y no a "generar desarrollo" o bienestar para sus pueblos. Incluso más, al no estar constituida la profesión de economista, existían muy pocas personas con formación sobre estos asuntos, siendo los temas económicos abordados de modo político, cultural o humanitario, y no de manera autónoma, disciplinaria o especializada. Es de notar que la constitución de la profesión es posterior a la independencia y se realiza, como en ALC, sólo en los años 50 , es decir cuando ya se ha producido la división entre la India y Pk. De hecho existe un quiebre generacional y epistemológico entre el pensamiento producido entre 1920 y 1950 y parte del producido entre 1950 y 1980. El quiebre generacional es obvio, el quiebre epistemológico proviene de la especialización, de la creación de una institucionalidad de las ciencias económico-sociales antes inexistente, de la gran cantidad de gente que estudia fuera de los países, de la inmensa circulación de ideas nuevas a través de redes muy activas de cientistas económico-sociales; todo lo cual otorga una autonomía "técnica" y "secularizada" al discurso de las ciencias económicosociales en la región. Ello, por cierto, no quiere decir que no hubiera unas pocas personas, antes de la independencia, que no fueran capaces de manejar el discurso económico más o menos especializado, como Debendra Kumar Das y Mohadev Ranade.

Respecto de Pk, el discurso de M. Iqbal, M. Jinnah o A. Maududi, por señalar 3 pensadores claves, entre 1920 y 1950 se encuentra fuertemente marcado por la cuestión nacional, por la autonomía musulmana en una región predominantemente hindú, por la cohesión y defensa de su religión y cultura, por la constitución de un estado independiente del poder colonial y de la mayoría no-musulmana. Las reiteradas alusiones al Corán, a la religión, a la autonomía cultural aluden sólo muy tangencialmente a aspectos económicos. Esto produjo lo que podría caracterizarse como la existencia de una mirada "culturalista", o en todo caso "politicista" en el discurso, casi ignorando la dimensión económica, que en otros momentos o lugares ha sido tan decisiva, llegándose a identificar cuestión pública con economía o discurso político con discurso sobre política económica.

Esta prescindencia del tema económico, o de su abordaje casi únicamente de manera tradicional, como justicia o preocupación por el pobre y el desvalido, permite que una vez establecida la profesión pueda desenvol- 
verse un pensamiento económico en un territorio casi intocado, es decir, con gran autonomía, respecto de los discursos tradicionales. Discusiones sobre la planificación, los planes quinquenales, desarrollo/subdesarrollo, precios de las materias primas, inflación, etc., no atañen al Islam y el criterio que se establece es de mucha libertad de opinión, mientras estas discusiones o ideas no "repugnen" al Corán.

Por lo mismo, al no existir un discurso económico suficientemente elaborado, a través de la larga duración, es casi imposible imaginar hibridaciones entre el pensamiento "profundo" y el pensamiento económico-social proveniente de ALC. De hecho, las hibridaciones producidas ocurrieron predominantemente con líneas de pensamiento presentes en autores formados en la escuela económica inglesa o anglosajona, más en general, y que eventualmente habían acogido elementos de la escuela soviética, y/o que se habían hecho eco de las renovaciones del socialismo árabe o bazismo (de ba'az o ba'ath) y/o del socialismo islámico, en parte debido a las irradiaciones del nasserismo.

\section{LA CIRCULACIÓN DE LAS IDEAS ENTRE ALC Y PK}

De buenas a primeras puede resultar sorpresiva la formulación acerca de la recepción y reelaboración de ideas provenientes de ALC en Pk. Pero esta sorpresa debería ir cediendo en la medida que se ha ido asumiendo que en los años 60 ALC se transformó en una región que irradió (exportó) numerosas ideas hacia el mundo y que hubo importantes redes a través de las cuales esas ideas encontraron nichos en África, Asia y Europa (ver Devés, 2008). En todo caso, diferentes autores se han referido al impacto que tuvieron las ideas procedentes de ALC en el SPI. Por ejemplo, Gustav Ranis destaca que es interesante notar "que el modelo de W. A. Lewis y su descendencia continúa siendo visto como relevante en el Sur y considerado una valiosa guía para las políticas, en lugares como China, India, Bangla-Desh, América Central e incluso en algunas partes del África Sud-sahariana" (2004: 5). Por su parte, Yogender Kumar Alagh, rector de la Universidad Jawaharlal Nehru en 1995, inaugurando un acto organizado por el Centro de Estudios Hispánicos de esa universidad, recordaba que le era familiar la obra de Furtado, "porque comprendió el meollo del problema del mundo subdesarrollado y su lazo con el mundo desarrollado", así como la obra de Prebisch y los dependentistas, para destacar a continuación que "durante los años 70 se hizo mucho trabajo en los países latinoamericanos sobre la

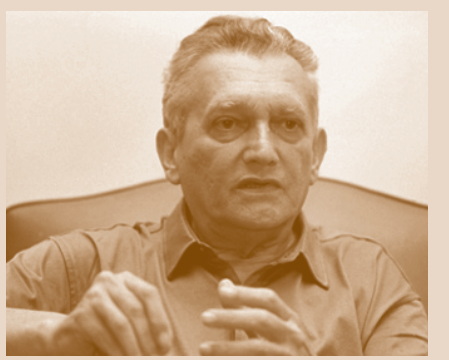

C. Furtado 
auto-dependencia que estudiábamos en nuestros programas académicos" y que hubo "discusiones sobre estas ideas", de modo "que tuvieron un impacto, por cierto" (Yogender Kumar Alagh 1997: VIII).

Ahora bien, ¿cuáles fueron, más específicamente, las maneras en que la producción de las ciencias económico-sociales de ALC llegó a los autores de Pk? Debe aclararse, en todo caso, que el objetivo no es determinar si estas ideas influyeron o no en las políticas, sino cómo fueron recepcionadas y reelaboradas por autores pakistaníes.

En otro trabajo se ha expuesto las maneras en que las ciencias económico-sociales de ALC pasaron a Asia y África (ver Devés, 2008). Allí se marcaron una serie de modelos de circulación a través de: Instituciones académicas del Primer Mundo; encuentros de cientistas económico-sociales; Redes sobre cuestiones del desarrollo; estadías de latinoamericanas en Asia y África; estadías de africanos y asiáticos en ALC; conferencias y congresos internacionales; $y$ publicaciones.

Para el caso de Pk deben, entre éstos, ponerse en relieve los siguientes:

a) Los contactos entre cientistas económico-sociales de ambas regiones, sea en visitas recíprocas, sea en reuniones de organismos internacionales donde trabajaban en conjunto, sea en centros académicos donde eran colegas o estudiantes. Por ejemplo, Mahbub ul Haq participó en diversas reuniones y grupos de trabajo con cientistas económico-sociales de ALC y no sólo esto sino que también organizó actividades en conjunto con aquéllos. En este sentido, es particularmente importante tener en cuenta las reuniones del Foro Tercer Mundo en 1973 en Chile y en 1975 en Karachi, donde se dieron cita cientistas económico-sociales de Chile, Pk y otros países, entre los cuales India, Bangla-Desh y Sri Lanka (ver Devés, 2006), como también la Conferencia Sur-Sur, realizada en Pekín en 1983 (ver Gauhar, 1983).

b) La adopción de estas ideas por autores renombrados, particularmente del Primer Mundo, que se ocuparon de difundirlas en regiones del Tercer Mundo, a través de misiones y asesorías o a través de cursos y artículos que impactaron en discípulos de esas regiones. Debe mencionarse a este respecto al Institute for Development Studies de Sussex University, donde las ideas económico-sociales de ALC tuvieron una importante presencia y adonde concurrieron economistas del SIP para realizar estudios, investigaciones y docencia.

c) El conocimiento de tales ideas a través de publicaciones, principalmente de revistas sobre cuestiones económico-sociales de orientación tercermundista que florecieron en los 60 y particularmente en los 70. Por 
ejemplo, Mahbub ul Haq participó en varias de esas revistas como Development Dialogue, Third World Quarterly, World Development, e International Development Review.

\section{TENDENCIAS DEL PENSAMIENTO ECONÓMICO-POLÍTICO EN PAKISTÁN EN LOS 60 Y 70}

Se pondrán en relieve 2 ó 3 tendencias eidéticas (en este caso, 2 ó 3 ideologías) que se encontraban presentes en el $\mathrm{Pk}$ de esos años y que sirvieron de marco, o de medioambiente, en el cual fueron recepcionadas las ideas económico-sociales de ALC. Se trata del "planificacionismo" y del "islamismo político", que puede dividirse en "socialismo islámico" (hacia la izquierda) e "islamización del Estado" (hacia la derecha).

\section{a) El planificacionismo}

Luego de la independencia y antes de la división entre Pk (Occidental) y Bangla-Desh (Pk Oriental), tan tempranamente como en 1948, fueron creados varios organismos como el Development Board, el Planning Advisory Board y un Comité Económico del Gabinete y poco más tarde, una Comisión de Planificación, mostrando que las ideas sobre el desarrollo y planificación eran las oficiales del recién nacido Estado. Inmediatamente se comenzó a elaborar y, muy pronto, a poner en práctica un Programa Sexenal de Desarrollo y unos años más tarde un primer y segundo Plan Quinquenal de Desarrollo.

Estas iniciativas se sustentaron en el ejemplo de los países de la Europa Oriental y en las ideas de algunos de sus economistas, como también en las inspiraciones de muchos economistas residentes en el mundo capitalista que sustentaban ideas "planificacionistas" de diverso pelaje y calibre, ideas que se hicieron muy hegemónicas en la segunda posguerra e inspiraron a los economistas profesionales y a muchos políticos de $\mathrm{Pk}$, la India y Sri Lanka e incluso, ya en los 70, a Bangla-Desh. Ello no debe ocultar, sin embargo, que en la redacción del primer plan de Pk, por ejemplo, tuvo mucha importancia el Harvard Advisory Group, que trabajó allí entre mediados de 1950 y 1970 aproximadamente, llegando a mediados de los 50 a contar con 17 profesionales en $\mathrm{Pk}$ (Rosen, 1985: 153).

La idea clave de los planes quinquenales en Pk consistió en que el Estado debía elaborar y llevar a cabo un programa de desarrollo que comprome- 
tiera a los diversos sectores de la economía. Aunque había claramente una noción de Estado económicamente activo, con propiedad pública y con inversión, ello no apuntaba necesariamente a debilitar el sector privado, ni expropiaba bienes de particulares. Tampoco enfatizaba necesariamente en cuestiones como justicia social, igualdad o reformas sociales radicales, aunque eso sí cambios económicos importantes. El principal objetivo del Programa Sexenal, a comienzos de los 50, fue formulado para alcanzar un crecimiento del 15\%, mejorar la situación de la balanza de pagos, crear nuevas oportunidades de trabajo, favorecer el progreso proveyendo educación, salud y otros servicios sociales y acrecentando los recursos técnicos y organizaciones del país (Mannan, 1970: 39).

\section{b) El islamismo político}

El islamismo político ha comprendido diferentes tendencias, desde mucho antes de la aparición de Pk como Estado y, por cierto, no sólo en el subcontinente. Sin embargo, el islamismo político desarrolló dos tendencias específicas que, aunque poseen conexiones con el pensamiento islámico de otras regiones, han sido caracterizadas por los especialistas como tendencias propias: se trata del "aligarhismo", proveniente de la escuela de Aligarh, y el "deobandismo" proveniente de la escuela de Deoband. El primero, tratando de abrirse al pensamiento y a los descubrimientos científicos europeos, el segundo afirmándose en la sola fuerza del Corán y de la Sunna (los ejemplos del Profeta) como bases. Las tendencias democráticas, liberales y socialistas, en el marco del Islam del subcontinente, se desarrollaron claramente en el marco de la tradición "aligarhista", que fue más proclive a la posición centralitaria (ser como el centro). Ambas posiciones se definieron como islámicas y ambas propusieron un pensamiento político.

\section{Socialismo islámico}

El principal representante, en la época que nos interesa, del socialismo islámico en Pk fue Zulfikar Bhutto, líder político y Primer Mandatario entre 1970 y 1977. Según Bhutto, Pakistán no podía progresar bajo el presente sistema económico social, ni podía llegar a ser un país fuerte, si la pobreza y la miseria seguían creciendo. Sólo puede progresar un país si el pueblo progresa (s/f: 5). Un país con un pueblo económicamente atrasado no puede nunca progresar (s/f: 11). Nuestro empeño deberá apuntar entonces a fortalecer al país a través de la democracia (s/f: 19). Los problemas básicos 
son los problemas económicos, de la pobreza y la miseria (s/f: 23). Siendo la igualdad la base del Islam y si la democracia no está contra el Islam, entonces ¿cómo la igualdad, que es llamada 'socialism' en inglés, puede estarlo? (s/f: 5). El manifiesto del Partido del Pueblo Pakistaní (Pakistan Peoples Party) está basado en el socialismo islámico que significa igualdad islámica (s/f: 23). La cuestión es ¿qué es socialismo? No es nada más que igualdad islámica ( $\mathrm{s} / \mathrm{f}: 30)$. El socialismo islámico no es ajeno al Islam. No está en conflicto con nuestras creencias. Si la democracia parlamentaria británica no está en conflicto con el Islam, yo no entiendo cómo los valores de igualdad y hermandad, que el Islam ha dado al mundo, podrían ser opuestos al Islam (s/f: 158). Socialismo islámico significa que nosotros queremos tener una economía socialista de acuerdo a nuestras condiciones, respaldada por nuestra propia herencia, emergiendo desde nuestras propias tradiciones y en conformidad con nuestros valores e ideología. Sólo este tipo de estructura económica puede proveer de iguales oportunidades para todos, proteger al ciudadano de la explotación y remover las barreras entre las clases privilegiadas y las clases explotadas. El socialismo es el interés directo de $\mathrm{Pk}$, un país subdesarrollado, destruido por la explotación interna y externa (s/f: 158).

Argumenta Bhutto que las raíces del socialismo se apoyan en una profunda visión ética de la vida. El Partido del Pueblo Pakistaní afirma seriamente que los altos ideales del Islam en relación con la sociedad pueden alcanzarse únicamente a través de un sistema socialista, aboliendo la explotación del hombre por el hombre. Creemos que la naturaleza de la justicia en el mundo exigida por nuestra religión es inherente en la concepción de una sociedad sin clases. En esto el Islam difiere fundamentalmente de otras religiones. El Islam no reconoce castas. La sociedad capitalista tiene una estructura de clases que es opuesta a la igualdad y hermandad prescrita a los musulmanes por el Islam (s/f: 158). En todo caso y por esto mismo, afirma que no es el primero en levantar el eslogan del socialismo islámico, Quaidi-Azam (M. A. Jinnah) fue el primero en usar este término y recordemos que él fue uno de los que fundaron este país. Fue él quien declaró que el socialismo islámico debe ser establecido en $\mathrm{Pk}$ (30). Fue él quien dijo que "Pk debe estar basado en firmes cimientos de justicia social y socialismo islámico" (s/f: 157). Por su parte, Liaquat Ali Khan dijo en 1949: "Existe una cantidad de ismos de los cuales se habla en la actualidad, pero estamos convencidos que para nosotros existe un solo ismo, llamado Socialismo Islámico" (s/f: 157).

Para producir un cambio en la situación internacional de Pk, es impera-

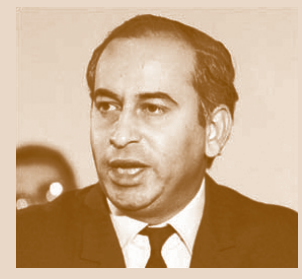

Z. Bhutto

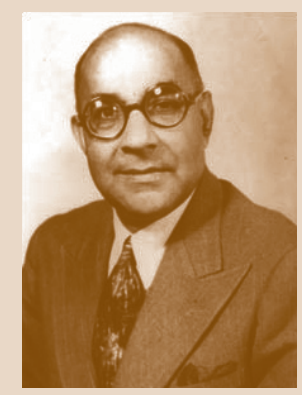

L. A. Khan 
tivo rechazar el presente sistema capitalista y reemplazarlo por un socialismo islámico. Sólo mediante este sistema autóctono (indigenous system), a partir de nuestra propia voluntad, seremos capaces de desterrar la pobreza (s/f: 157). En un discurso pronunciado el 25 de febrero de 1970 sostiene que han pasado veintidós años desde la Independencia, pero el pueblo no ha visto ninguna prosperidad. Cuán miserable es una gran cantidad del pueblo. Nosotros queremos detener esta explotación y liberarte de tus cadenas. Queremos una atmósfera de libertad para el desarrollo de nuestra personalidad nacional (s/f: 24-25).

De hecho, argumenta, quienes están contra el socialismo islámico ahora, son los mismos que estuvieron contra el rey Amunullah Khan en Afganistán, contra Ataturk en Turquía, contra Nasser en Egipto, y contra Sukarno en Indonesia, y sobre todo contra el Quaid-i-Azam y el Quaid-i-Millat (s/f: 30).

$\mathrm{Al}$ referirse a la autodeterminación de los pueblos, señala que no habla sólo por Pk sino por un principio básico que afecta a Asia, África y Latinoamérica (s/f: 234). En su discurso ante el Consejo de Seguridad de las Naciones Unidas, el 12 de diciembre de 1971, se refiere a la presencia allí de representantes de otros estados miembros de África y Latinoamérica. Pk es parte del Tercer Mundo y cuando ha estado en nuestras manos hemos tratado siempre de contribuir a la causa del Tercer Mundo (s/f: 258). Todos venimos de la misma fraternidad, somos todos compañeros de armas. Hemos sido explotados, hemos sido subyugados, somos todos víctimas de la explotación sin piedad, de ahí esta solidaridad en el principio de la no-intervención, de la autodeterminación y de la coexistencia pacífica (s/f: 240). No debemos pensar solamente en términos de nuestro propio país. En tanto que asiáticos y como miembros del Tercer Mundo, no debemos renunciar nunca a la autodeterminación (s/f: 242).

\section{Islamización del Estado}

La segunda fórmula de islamismo político y la tercera ideología con relevancia para lo que interesa en el Pk de la época fue claramente menos focalizada a cuestiones económicas y con mayor énfasis en lo político-cultural o político-religioso. Se trató del islamismo radical, que apuntaba a transformar a Pk en un Estado confesional islámico, como una cuestión no sólo de hecho sino también de derecho.

Aunque claramente menos sensible a cuestiones económico-sociales, esta tendencia no puede permanecer al margen completamente de la dis- 
cusión o de la preocupación económico-social, que se hace tan patente en la época. La más importante organización propulsora de estas ideas fue el partido Jamat-e-Islami (o "Jama'at-i-Islami", en otras versiones) fundado e inspirado ideológicamente por Abul Ala Maududi (o Mawdudi). Según $\mathrm{K}$. Bahadur la formulación de un sistema económico islámico ocupa un lugar importante en las ideas de Maududi, dividiendo los sistemas existentes en capitalista, comunista e islámico. Su crítica al capitalismo está principalmente basada en el problema del interés al dinero, prohibido por el Corán, y más ampliamente en la idea que el capitalismo ha adulterado los principios naturales de la economía $(1977,179)$. Su crítica al comunismo se orienta hacia lo que se ha llamado el "economicismo" o la idea que el comunismo considera todas las otras dimensiones únicamente desde el punto de vista económico, es decir, que la metafísica, la ética, la historia, la ciencia y las humanidades están todas influidas por el punto de vista económico. Por otra parte, sostiene que por destruir el capitalismo el sistema comunista ha creado un gran Estado capitalista (1977, 185-6). Piensa que el sistema islámico sigue una vía intermedia entre el capitalismo y el comunismo, siendo por lo demás ambos productos de la civilización occidental. Las diferencias entre comunismo, capitalismo y fascismo (una forma aguda de capitalismo) son menores. Las 3 tienen una perspectiva cultural similar y se refieren a que los seres humanos no deben necesariamente obedecer a Dios $(1977,186)$.

En 1970, el partido en su 'Manifiesto de enero' se opone al latifundismo y al capitalismo occidental. Los postulados básicos de su programa han sido resumidos como "una justa distribución de la riqueza, la prevención de la concentración de la riqueza en pocas manos, terminar con la opresión y la explotación en sus variadas formas, igualdad de oportunidades para todos los ciudadanos, eliminación de la pobreza asegurando que nadie carezca de las satisfacciones básicas para la vida. Propone la limitación de la propiedad de la tierra a cantidades que no superen los 200 acres y apoya la nacionalización (compensada) de interés nacional. Se opone a carteles y monopolios y propone organizar la banca de acuerdo a criterios islámicos (Syed, 1982: 120-1).

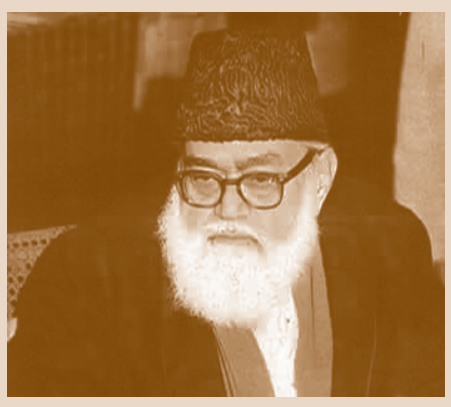

A. A. Maududi

\section{ALGUNAS PERSONAS QUE ACOGIERON LA PRODUCCIÓN}

DE ALC

Son numerosísimos quienes en el SIP que conocieron y/o se inspiraron en el pensamiento procedente de ALC. Es el caso, en la India, de Jagdish Bha- 


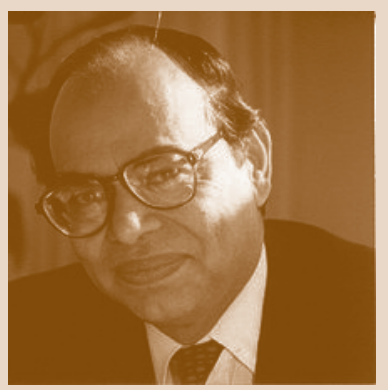

M. ul Haq gwati (1982), Padma Desai (1973), R. Narayanan (1983), Ashutosh Varshney (1981) y Devendra Thakur (1987); en Sri Lanka, de Gamani Corea (1992), Satchi Poonambalam (1981), Susantha Goonatilake (1975a, 1975b) y Asoka Barandage (1983); en Bangla Desh, de Nurul Islam (2003) y Emajudin Hamed (1980); en Pakistán, de Hamza Alavi (1983), Altaf Gauhar (1983), Akmal Hussain (1988), Mohammed Hussain (1987), K. M. Azam (1988) y, sobre todo, Muhammad Abdul Mannan y Mahbub ul Haq, en quienes se focalizarán las páginas siguientes. Ello debido a que estos dos autores pakistaníes se han detectado como "receptores" y "reelaboradores" relevantes del pensamiento económico-social de ALC, más por el interés de sus casos para los estudios eidológicos, debido a la originalidad de sus reelaboraciones y a las inmensas diferencias con que cada uno procesó los insumos de ALC para elaborar sus propios sistemas eidéticos, que por la importancia para su pensamiento de las inspiraciones que les llegaron desde ALC.

a) Una economía para la liberación y el desarrollo humano: Mahbub ul Haq

M. ul Haq (1934-1998), oriundo de Cachemira, se formó en Cambridge, Yale y Harvard, fue director de la Oficina de Planificación de Pk en los 60 y luego se desempeñó en el Banco Mundial. Fue uno de los creadores de la noción "desarrollo humano" y uno de los inspiradores del Informe sobre Desarrollo Humano del PNUD, que contribuyó a poner en marcha en $1990^{4}$.

Por otra parte, M. ul Haq fue uno de los pioneros en la promoción del encuentro y el diálogo entre cientistas económico-sociales de Asía, África y ALC, cosa que se expresó, por ejemplo, en la co-organización del Foro Tercer Mundo (FTM), cuya reunión preparatoria se realizó en Santiago de Chile en abril de 1973 y cuya primera reunión se realizó en Karachi, en enero de 1975. Ul Haq es quien más teorizó sobre el sentido y proyecciones de este tipo de iniciativas y para ello echó mano, entre otros insumos, de las ideas procedentes de ALC.

En La cortina de la pobreza (The Poverty Curtain) dedica una parte importante a esclarecer la evolución de las ideas sobre el desarrollo y cuáles han sido sus propias evoluciones a este respecto, teniendo en cuenta, por una parte, su formación en temas del desarrollo y, por otra, su interpreta-

\footnotetext{
${ }^{4}$ Sus trabajos más importantes fueron: The Strategy of Economics Planning (1963) y The Poverty Curtain (1974).
} 
ción respecto de sus propias experiencias en la tarea de planificación del desarrollo en $\mathrm{Pk}$, cosa que tuvo que ver particularmente con la concentración de la riqueza, en sectores sociales y en regiones geográficas, como producto del crecimiento económico del país y del modelo de crecimiento y planificación que se había aplicado (1976: 5-6). Es en el marco de esta reflexión donde Ul Haq incorpora la reflexión proveniente de ALC, particularmente algunas ideas del dependentismo (1976: 57-58). Cuestionando los modelos de desarrollo utilizados, se ocupa de la poca importancia que se le ha dado a la distribución del ingreso, a la disminución de la pobreza en sus formas extremas, a la disminución del desempleo y del subempleo. Ante esto recoge algunas ideas de Dudley Seers, autor fuertemente inspirado en el pensamiento de ALC (1976: 36), pero sobre todo acude a la experiencia de China, pues le parece que ese país ha obtenido importantes avances en la disminución del analfabetismo, de la extrema pobreza y del desempleo, focalizándose en estos asuntos más que en el "crecimiento" (1976: 37). Estas ideas le llevan a sostener que debe reevaluarse (y disminuirse), en las estrategias de desarrollo, la importancia atribuida al comercio internacional, sobre todo en las concepciones convencionales. Es precisamente en este punto que recoge las ideas provenientes de ALC, particularmente aludiendo a Celso Furtado y a Theotonio dos Santos, como a las propuestas del FTM (1976: 57-58). Esto es muy relevante para Ul Haq, pues es a partir de allí que va a plantearse la constitución de una red y de una institucionalidad de la intelectualidad de las regiones periféricas para hacer frente al subdesarrollo, teniendo en cuenta ahora la producción intelectual de esta propia intelectualidad, mucho más que los aportes que vienen desde fuera y que están conduciendo a formas perversas de desarrollo. Sobre la base de estas ideas es que Ul Haq se transforma en uno de los principales, sino el primero, de los ideólogos del FTM.

La imagen de sí mismos que proponen quienes se reúnen en el FTM puede armarse sobre la base de algunas ideas que son los pilares que afirman este discurso5: 1) El discurso del Tercer Mundo se alimenta de concepciones del desarrollo que han sido elaboradas por la intelectualidad del mundo desarrollado y que son inadecuadas (Declaración de Santiago). 2) Las ideas e ideologías para el Tercer Mundo deben venir desde dentro (Desai, 1973: 63). 3) Para ello debe hacerse investigación que sea re-

\footnotetext{
${ }^{5}$ Utilizo aquí algunos trozos del trabajo Devés (2006) "Los cientistas económico sociales chilenos en los largos 60 y su inserción en las redes internacionales: la reunión del Foro Tercer Mundo en Santiago en abril de 1973". Universum, № 9, septiembre.
}

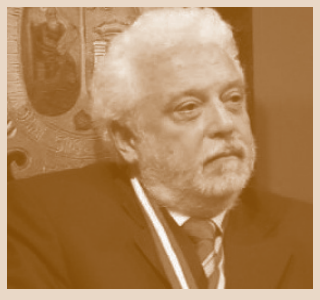

T. dos Santos 
levante-pertinente (Declaración de Santiago) o, en otras palabras, deben establecerse instituciones de autosuficiencia intelectual (Comunicado de Karachi). 4) Esto deberá ser financiado por un fondo fiduciario, del orden de los mil millones de dólares (Comunicado de Karachi). 5) Los cientistas económico-sociales del Tercer Mundo deben tener una plataforma donde encontrarse para intercambiar ideas (Declaración de Santiago). 6) Los intelectuales del Tercer Mundo deben organizarse en torno a las inquietudes (formuladas en el "Comunicado") en vistas a crear un ambiente propicio para un orden nacional e internacional equitativo (Comunicado de Karachi $\left.^{6}\right)$ o, dicho en otras palabras: los intelectuales deben buscar los modos de transmitir sus ideas en los corredores del poder del Tercer Mundo (Desai, 1973: 63). 7) De modo que pueda generarse una revolución intelectual (Declaración de Santiago) que debe ser llevada a cabo en cada universidad, instituto y foro donde se discuta sobre el Tercer Mundo y que el "Comunicado de Karachi” formula así: es necesario librar una revolución intelectual permanente para superar la dependencia del Tercer Mundo e introducir cambios profundos en el orden interno y externo que los países en desarrollo encaran actualmente.

Estas ideas básicas fueron explicitadas, desarrolladas o complementadas en diferentes documentos. Los "Estatutos" del FTM aprobados en Karachi en 1975 se abren con un "nosotros, los expertos en ciencias sociales y otros intelectuales del Tercer Mundo deseosos de hacer un aporte más constructivo y significativo a los pueblos de nuestros países, estamos convencidos de que por ahora la mejor forma de lograrlo es mediante la fundación, organización y respaldo de un FTM" (Constitución, 1975: 1). Las funciones principales del Foro, según este documento son: proporcionar una plataforma para el intercambio de puntos de vista; proporcionar apoyo intelectual a los países del Tercer Mundo; estimular y organizar investigaciones socioeconómicas; prestar asistencia a las respectivas entidades; respaldar los programas de cooperación entre los países en desarrollo; y exponer opiniones sobre cuestiones internacionales que afectan al Tercer Mundo (Estatutos, 1975: 1-2).

Avanza en el desarrollo de algunas de estas ideas Mahbub ul Haq, participante y promotor de las reuniones de Santiago y Karachi y presumible-

\footnotetext{
${ }^{6}$ En dicha reunión participaron las siguientes personas procedentes de ALC: F. H. Cardoso, Celso Furtado, Helio Jaguaribe, Enrique Iglesias, Amilcar Herrera, Enrique Oteiza, Oscar Arias, Luciano Tomassini, también Oscar Pino Santos, Manuel Pérez Guerrero, Santiago Macario, José M. Suárez Mier y Otoniel Velasco. Hubo igualmente figuras de Asia y África que ya conocían la producción de las ciencias económico-sociales de ALC, como Samir Amin de Senegal, Gamani Corea de Sri Lanka, Mahbub ul-Haq de Pakistán y Justinian Rweyemamu de Tanzania.
} 
mente uno de los redactores de los documentos evacuados por éstas. Presentando específicamente el "Comunicado de Karachi", argumenta que uno de los objetivos es "equipar a nuestros negociadores (del Tercer Mundo) en conferencias internacionales con ideas poderosas y escritos concretos que puedan ser una voz de unión para el Tercer Mundo (1975: 9). De hecho, hemos vivido de conceptos y pensamientos en gran parte tomados de Occidente y nuestro propio pensamiento ha sido frecuentemente juzgado con los estándares occidentales (1975: 9). Nuestras ideas no han alcanzado aceptación porque han sido juzgadas con estándares inadecuados y no hemos sido capaces de organizar foros para proyectar nuestro pensamiento (1975: 10). Ul Haq señala estar firmemente convencido que nuestra independencia nacional no es completa si la liberación política no es seguida de liberación económica e intelectual (1975: 10), y la liberación intelectual es la más difícil de alcanzar, dado que muchos de nosotros somos prisioneros de nuestro pasado educacional. En un amplio sentido entonces, el FTM constituye un movimiento de autosuficiencia (self-reliance) intelectual. Ello quiere decir que a nivel nacional significa la búsqueda de soluciones propias dentro del propio sistema de valores y a nivel internacional es un esfuerzo por organizar intelectualmente al Tercer Mundo, para combatir por sus justas demandas en todos los foros internacionales. Esto es tomar los dos principales temas de nuestra agenda, NOEI (Nuevo Orden Económico Internacional) y desarrollo nacional, en sentido muy específico y realista (1975: 10).

\section{b) La planificación en el socialismo islámico: Muhammad Abdul Mannan}

Muhammad Abdul Mannan nació en 1938 en Bengala Oriental (hoy Banglad-Desh). Luego de recibir su Master on Economics por la Universidad Rajshahi en 1960 sirvió en varias reparticiones económicas del gobierno. En 1970 fue a USA, a la Michigan State University, por su M. A. Economics y luego realizó allí el doctorado. Obtenido éste, enseñó en Papúa Nueva Guinea, y en 1978 se incorporó al Centre for Research in Economics, de Yeddah, posteriormente llamado Center for Research in Islamic Economics. Vivió y trabajó en Pakistán Occidental (hoy Pakistán), antes de la separación de Bangla-Desh ${ }^{7}$. Junto a M. Siddiqi, es uno de los autores más ampliamente citados en el tema de la economía islámica.

\footnotetext{
${ }^{7}$ Algunas de sus publicaciones han sido: Islamic economics: theory and practice. Boulder: Westview Press, 1987, c1986; Economic development and social peace in Islam: an analytical study of
} 
Mannan escribió un trabajo (un manual para estudiantes) sobre Problemas económicos y planificación en Pakistán, que tuvo varias ediciones, siendo la de 1970, la 4a a la que se utiliza en este caso. Algunos de los capítulos de la obra se refieren a los siguientes asuntos: Planeación, Planeación en Pk, Sectores estratégicos de la economía de Pk, Desarrollo regional en Pk, Desarrollo económico, Sistemas económicos comparados (capitalismo, socialismo, comunismo, fascismo y socialismo islámico).

El problema que se plantea es acerca de la economía aplicada al bienestar y el papel que puede jugar allí la planificación y el Estado. Su marco de referencia principal es la escuela económica inglesa, como lo prueban sus referencias iniciales a A. C. Pigou y J. M. Keynes, especialmente en la subdisciplina de los estudios del desarrollo, cuya inspiración le viene, y esto es lo particularmente importante, del caribeño W. A. Lewis. Por cierto, ello no quiere decir que no haga referencia a economistas de otras escuelas y procedencias, como W. W. Rostow y F. von Hayek, por un lado, y O. Lange por otro, y también a otros economistas del desarrollo como A. O. Hirshman y G. Myrdal. Mannan echa mano igualmente a autores indios y pakistaníes, particularmente a su compatriota M. ul Haq, de quien también se ocupa el presente artículo. Las referencias a W. A. Lewis son particularmente frecuentes, enmarcándose su lectura en la teoría de la modernización. Lewis y Rostow marcan un claro sesgo en la obra de Mannan, aunque al final del libro, cuando se ocupa del socialismo islámico, pueda dejar de lado varios de los criterios fijados a lo largo del libro. A diferencia de M. ul Haq, las citaciones de Lewis que hace Mannan son las de alguien que se ocupa de desarrollo y planificación, pero no asumiéndolo como autor que provendría o que hablaría para una experiencia similar a la de Mannan por ser, por ejemplo, ambos provenientes del Tercer Mundo. No puede decirse que en Mannan haya algo así como una conciencia del Sur, tercermundista o periférica, como la hay en otros autores africanos, asiáticos o latinoamericanos.

Su Problemas económicos y planificación en Pakistán termina con un repaso de los diferentes sistemas vigentes: socialismo, capitalismo, comunismo, fascismo, ocupándose finalmente del socialismo islámico, que define del modo siguiente: El socialismo tal como es concebido por el Islam está principalmente basado en las enseñanzas del Santo Corán y en las palabras

the process of economic development in the Muslim community of today (with an account of real-life experiments in Bangladesh). 1st ed. London: Ta Ha Publishers; Dhaka, Bangladesh: Bangladesh Social Peace Foundation, 1990, c1989; Economic development in an Islamic framework; Growth and development of small enterprise: the case of Bangladesh. 
y prácticas del santo Profeta. No es capitalista ni comunista, está asentado sobre sí mismo y combina los rasgos positivos de una sociedad sana y balanceada. Las bases fundamentales de la democracia islámica o socialismo, conceptos que son intercambiables, se afirman en los versículos del Corán (1970: 435-6). Según Mannan, el socialismo islámico está basado en la igualdad social, cuestión que fundamenta en el hecho que "la marca distintiva del Islam es su respeto por la individual y absoluta igualdad humana. Todos gozan de igual estatus social, política y económicamente. El estatus de las mujeres, menores de edad y esclavos en el Islam es casi de igualdad y proverbial. La dignidad del trabajo es un artículo de fe en el Islam" (1970: 437).

La moderna concepción de la planificación, que se refiere a la utilización de los recursos del país para alcanzar ciertos objetivos, es reconocida por el Islam. Planificación económica en el Islam no es otra cosa que una síntesis de planificación por inducción y por dirección, que son las dos opciones que ha señalado en la planificación contemporánea. La cooperación entre el sector público y el privado es la base de la planificación económica en el Islam (1970: 443). El socialismo islámico condena al capitalismo y la explotación en todas las formas, basado en los eternos principios de la justicia social y de la universal hermandad entre los hombres. Pk debe afirmarse sobre los cimientos seguros de la justicia social y del socialismo islámico (ningún otro "ismo") que enfatiza la igualdad y hermandad entre los hombres (1970: 444).

Mannan se fue transformando en uno de los más importantes referentes en una línea de pensamiento que se ha denominado la "economía islámica”, disciplina que se ubica en la intersección entre el quehacer económico y el teológico, pero donde curiosamente Mannan no ha considerado sus temas originales como desarrollo y planificación, volcándose en cambio a otras cuestiones, como son los aspectos filosóficos de la economía islámica, la economía islámica como ciencia social, las cuestiones de lo individual, lo social y lo estatal, el orden económico islámico y el mercado (1970: 1); abandonando, por otro lado, sus nociones acerca de socialismo y/o democracia islámica, cosa que se hace más explicable al considerarse que esta línea de trabajo la ha desarrollado principalmente en Arabia Saudita. En otras palabras, los insumos provenientes del pensamiento de ALC, y en general de las teorías o escuelas del desarrollo, dejaron de serle útiles para su desarrollo profesional posterior. 


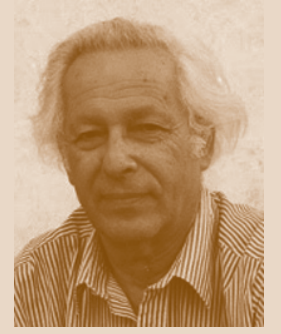

S. Amin

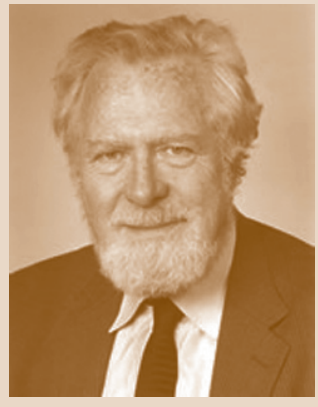

D. Seers

\section{CONCLUSIONES Y PROYECCIONES}

Puede concluirse, entonces, respecto a los dos planos de que se ocupa este trabajo: el de la recepción y el de la reelaboración, lo siguiente:

5.1. Sobre la recepción: Vistas algunas de las obras de los pakistaníes estudiados, puede afirmarse que los trabajos de W. A. Lewis, Celso Furtado, Theotonio dos Santos fueron conocidos y que también fue conocida la obra de otros autores, de procedencia no latinoamericana pero con presencia en su propia obra de las ideas provenientes de ALC, como Samir Amin o Dudley Seers. Para ninguno de los dos autores de Pk trabajados en este artículo, sin embargo, tales ideas tuvieron gran importancia, no ubicándose para ellos a nivel de las ideas clásicas sobre desarrollo ni del keynesianismo. De hecho, los conceptos más típicos como centro/periferia, deterioro en los términos del intercambio, industrialización por invitación y otros poco y nada aparecen, y en todo caso, no en referencia específica a su origen en ALC, como tampoco se establece una discusión respecto de la validez de tales conceptos, como se hace en África, por parte de Samir Amin (1981 y 1999), Colin Leys (1975 y 1996) o Dan Nabudere (1977a, 1977b y s/f).

5.2. Respecto a las reelaboraciones: Puede señalarse que, habiéndose asumido que en el marco del medioambiente intelectual de Pk se recibieron los organismos eidéticos de ALC, puede concluirse que éstos fueron hibridados en primer lugar con otros que provenían de líneas geneidéticas de procedencia inglesa (J. M. Keynes, A. C. Pigou) y de procedencia usamericana (W. W. Rostow), autores totalmente reconocidos en la discusión sobre desarrollo y modernización en la época. Pero, por otra parte, y esto es lo más relevante para el efecto, también fueron hibridados con algunos elementos procedentes de autores islámicos $\mathrm{y}$, particularmente, pakistaníes contemporáneos como Zulfikar Bhutto y (en el caso de Mannan) del propio Mahbub Ul Haq, y con otros elementos provenientes del pensamiento musulmán (también en el caso de Mannan).

Ahora bien, más específicamente, ¿cómo se produjeron las hibridaciones o los cruzamientos eidéticos y las reelaboraciones en los autores estudiados? En el caso de Mahbub ul Haq, éste insertó las ideas provenientes de ALC claramente en el marco de las ideas sobre desarrollo que circulaban en su época, sirviéndole específicamente para hacer la crítica de los paradigmas dominantes en los cuales él se había formado: Yale, Oxford y Harvard, como también en medio de lo cuales se había desenvuelto: el Banco 
Mundial y la Oficina de Planificación de Pk ampliamente asesorada por la Misión de Harvard. La clave de lo que pretende hacer Ul Haq es una suerte de revolución copernicana en las ideas sobre desarrollo, desde su focalización en el crecimiento hacia la focalización en la satisfacción de las necesidades básicas. Para ello debe hacer una crítica del desarrollismo clásico. En una tarea intelectual que tiene inspiraciones, parentescos y similitudes con el dependentismo, Ul Haq incorpora las ideas de ALC, asumiéndolas en el marco de esta reflexión tercermundista que trabaja en relación al FTM, como un think-tank de lo que podría llamarse la "intelectualidad orgánica del mundo periférico".

En el caso de M. A. Mannan, la incorporación de la obra proveniente de ALC, la de W. A. Lewis, es menos elaborada que en el caso de Ul Haq. La obra de Lewis le significó principalmente un material útil para redactar su manual sobre planificación económica, en un momento en que las más importantes tendencias de pensamiento del país y la región asumían la planificación como algo necesario. Este material le permitió construir, con cierto instrumental teórico, una versión algo más elaborada que sus predecesores o contemporáneos (Jinnah, Bhutto y otros) de un "socialismo islámico". Pero la versión del socialismo islámico de Mannan es "concordista" y "light", en la medida que incorpora diferentes elementos conceptuales sin realizar una verdadera síntesis, poniéndolos todos en un receptáculo y sin detenerse en la elaboración de un corpus teórico, contentándose con juntar los elementos sin construir una síntesis con suficiente identidad. Mannan acoge el "planificacionismo lewisiano", el socialismo islámico y las ideas de Maududi en conjunto, aunque en su desarrollo posterior se acercará claramente más a Maududi, no necesariamente por rebatir las otras ideas sino simplemente dejándolas fuera de su discurso.

En todo caso, luego de pocos años, Ul Haq y Mannan se desconectan de la trayectoria del pensamiento de ALC sobre cuestiones económico-sociales.

5.3. Otro elemento interesante de concluir es la comparación entre las recepciones y reelaboraciones del pensamiento procedente de ALC en los autores de Pk con casos ocurridos en África o en otros países del SIP. Los autores africanos o que trabajan en África o sobre África y que habían utilizado los insumos provenientes de ALC, tendieron a proyectar sus reflexiones radicalizando las propuestas de los autores de ALC: Samir Amin hacia el corte radical, mediante "desconexión", de los lazos de dependencia y mediante la interpretación de la economía africana como periférica respecto 
de la periferia latinoamericana y caribeña; Walter Rodney sumando a la explotación económica del centro la explotación por motivos raciales; Colin Leys realizando un crítica de los postulados dependentistas a partir de su propia radicalización y eventualmente de su reducción al absurdo. Los de $\mathrm{Pk}$, en cambio, van por otros caminos. Su opción más bien es prescindir del pensamiento originado en ALC, pasando a utilizar otros ingredientes para sus nuevos guisos eidéticos: el caso de ul Haq, muy ligado a las evoluciones del pensamiento global sobre desarrollo y a los organismos internacionales ocupados de éste; el caso de Mannan, desconectándose intelectualmente de los antecedentes provenientes de ALC y de toda la producción internacional sobre desarrollo para orientarse hacia la "economía islámica", más ligada a los tópicos convencionales de la economía clásica y a la teología islámica fundamentalista.

5.4. Sin embargo, si ul Haq o Mannan van por caminos diferentes, dejando también de lado la producción proveniente de ALC, no por ello todos los autores de Pk prescinden de ésta. De hecho, las líneas de trabajo de los 70 de que se ha ocupado este artículo se continúan durante los 80 , aunque sea por parte de otros autores pakistaníes, como Mohammed Hussain (1987) y K. M. Azam (1988), por ejemplo. Éstos continúan utilizando la obra de algunos autores de ALC, tanto como la búsqueda de un tipo de desarrollo que tenga en cuenta las necesidades y especificidades de los pueblos (según lo pretendió Ul Haq), como de un tipo de quehacer económico y una planificación que se articule a las ideas islámicas (según lo pretendió Mannan). Incluso estas dos líneas de reflexión convergen en algunos autores, como en el caso del recién citado Mohammed Hussain, quien recogiendo la obra de W. A. Lewis, pero también utilizando la obra de Ul Haq y Mannan, apunta a elaborar un tipo de planificación para el desarrollo que tenga en cuenta las condiciones de un estado islámico, realizando, por otra parte, elaboraciones teóricas que superan algunas de las anteriores.

5.5. La conclusión de un trabajo es, a menudo, el inicio de otro. En este caso, la conclusión en torno a la forma en que las ideas de las ciencias económico-sociales de ALC llegaron a Pk se ha topado con la pregunta de la manera en que estas ideas se cruzaron con otras y/o fueron reelaboradas. Por otra parte, aparece el problema de cómo se generan las nuevas ideas (o el conocimiento) en los medioambientes intelectuales.

Por cierto, cuando se habla aquí de generación de nuevas ideas no se está haciendo alusión a un asunto de tipo neurológico, psicológico y ni siquiera 
sociológico. En todo caso, se está más cerca de los desarrollos que ha hecho la historia de la ciencia, siendo el ámbito de trabajo los estudios eidéticos. El surgimiento de nociones como "desarrollo humano" y "economía islámica", respecto de los cuales no procede aquí discutir la validez, suponen una cierta revolución en los paradigmas. La aparición de nuevos sistemas de pensamiento supone la asunción de variados insumos eidéticos que se combinan de un modo específico en un medioambiente determinado.

Descubrir las "leyes" de aparición de estos nuevos organismos eidéticos y fomentar la creación de más y mejores productos eidéticos parecen ser dos de los más importantes quehaceres de los estudios eidológicos como disciplina. Esto se hace particularmente acuciante para un quehacer aplicado que quiere contribuir con urgencia al bien-pensar de los pobres del mundo, si puede decirse, de algún modo, continuando la trayectoria de Mahbub ul Haq.

\section{REFERENCIAS}

Alavi, H. (1983). "Class and state” en Gardezi, Hassan y Rashid, Jamil (edit) Pakistan. The roots of dictatorship. Delhi: Oxford U. Press.

Amin, S. (1981). La acumulación a escala mundial. Crítica a la teoría del desarrollo. $5^{\text {a }}$ edición ( $1^{\circ}$ en francés, 1970; Postfacio, 1971). México: Siglo XXI.

- (1999). Miradas a un medio siglo. Itinerario intelectual 1945-1990. Madrid: Plural-Iepala.

Azam, K. M. (1988). Economic and politics of development. An Islamic perspective. Karachi: Royal Book Company.

Bahadur, K. (1977). The Jama'at-i-Islami of Pakistan. Political thought and political action. New Delhi: Chetana Publications.

Bhagwati, Jagdish (1982). "W. Arthur Lewis: an appreciation" en Gersovitz, Mark et al. The theory and experience of economic development. Essays in Honor of Sir W. Arthur Lewis, Allen \& Unwin, Londres.

Bhutto, Z. A. (s/f). Marching toward democracy: statements, articles, speeches, Rawalpindi.

Corea, G. (1992). Taming commodity markets. The Integrated Programme and the Common Fund in UNCTAD, Vistaar Publications, New Delhi

Desai, P. (1973). "Third World social scientist in Santiago" en World Development, Vol. 1.

Devés, E. (2006). "Los cientistas económico sociales chilenos en los largos 60 y su inserción en las redes internacionales: la reunión del Foro Tercer Mundo en Santiago en abril de 1973". Universum, Vol. 21, № 1, 138-167. Disponible en: http://www.scielo.cl/scielo.php?pid=S0718-2376 20060001 00009\&script=sci_arttext [Consulta: 12.03.10]. 
(2008). "La circulación de las ideas económico-sociales de Latinoamérica y el Caribe en Asia y África ¿Cómo llegaron y cómo se diseminaron? (1965-1985)". Universum Vol. 23, № 2, 86-111. Disponible en: http://www. scielo.cl/scielo.php?pid=S0718-23762008000200005\&script=sci_arttext [Consulta: 20.06.11].

Devés, E. y Melgar-Bao, R. (2005). "El pensamiento de Asia en América Latina, Hacia una cartografía”. Revista de Hispanismo Filosófico No 10, 19-46.

FTM (Foro Tercer Mundo) (1975). "Cominicado de Karachi", en Carpeta Foro del Tercer Mundo. Santiago: Biblioteca CEPAL.

Gauhar, A. (1983). "Introduction" a The rich and the poor. Development, negociations and cooperation. An assessment. London: Third World Foundation.

Goonatilake, S. (1975a). "Development thinking as cultural neo-colonialism the case of Sri Lanka". IDS Bulletin, Vol. 7, No 1, Sussex University.

- (1975b). "Cultural dependence. The debate continues". IDS Bulletin, Vol. 7, No 3, Sussex University.

Hamed, E. (1980). Bureaucratic elites in segmented economic growth. Pakistan and Bangladesh. Bangladesh University Press Limited.

Hussain, Akmal (1988). Strategic issues in Pakistan's economic policy. Lahore: Progressive Publishers.

Hussain, Mohammed (1987). Developing planning in an Islamic state. Karachi: Royal Book Company.

Islam, Nurul (2003). Exploration in development issues. Selected essays of Nurul Islam. Burlington: Ashgate.

Leys, C. (1975). Underdevelopment in Kenya. The political economy of neocolonialism 1964-1971. Berkeley - Los Angeles: University of California Press.

Leys, C. (1996). The rise and fall of development theory, EAEP, Nairobi; Indiana University Press - Bloomington, James Currey - London.

Mannan, Muhammad Abdul (1970). Economic problems and planning in Pakistan. Lahore: Ferozsons.

__ (1977a). "Imperialism struggles in Tanzania”. UTAFITI, Vol. 2, № 1, Dar es Salaam.

(1977b). The political economy of imperialism. Dar es Salaam: Tanzania Publishing House.

— (s/f). www.aaps.co.zw/publications/global.html.htm [Consulta: 22.04. 2004].

Narayanan, R. (1983). "Latin American studies in India". Latin America Research Review, No 3, Austin, Texas.

Poonambalam, S. (1981). Dependent capitalism in crisis. The Sri Lankan economy 1948-1980. Colombo: Lake House.

Ranis, G. (2004). "Arthur Lewis' Contribution to Development Thinking and Policy". Disponible en: www.econ.yale.edu/ granis/ [Consulta: 13.08.2004].

Rosen, G. (1985). Western economist an Eastern societies. Agents of change in South Asia 1950-1970. New Delhi: Oxford U. Press y John Hopkins U. Press. 
Syed, A. H. (1982). Pakistan. Islam politics and national solidarity. New York: Praeger.

Thakur, D. (1987). Political economy of the Third World Countries. New Delhi: Deep and Deep Publicatons.

Ul Haq, Mahbub (1963). The Strategy of Economics Planning: a case study of Pakistan. Pakistan Branch: Oxford University Press.

Ul Haq, Mahbub (1976) [1974]. The Poverty Curtain. New York: Columbia U. Press. [(1978) La cortina de la pobreza. México: Fondo de Cultura Económica].

Varshney, A. (1981). "Political economy of Western aid to the Third World: a structural reinterpretation”. India Quarterly, Vol. XXXVII, july-september, No 3.

Yogender Kumar A. (1997). "Discurso del Rector de la U. J. N profesor Dr. Y.K.A. pronunciado en el acto inaugural de la conferencia", en Shyama Prasad Ganguly: Por los caminos del otro. Jornadas indias sobre las culturas española, portuguesa y latinoamericana. Nueva Delhi: Consejo Indio de Relaciones Culturales. 